\title{
Observation of the Spin Peltier Effect for Magnetic Insulators
}

\author{
J. Flipse, ${ }^{1,{ }^{*}}$ F. K. Dejene, ${ }^{1}$ D. Wagenaar, ${ }^{1}$ G. E. W. Bauer, ${ }^{2,3}$ J. Ben Youssef, ${ }^{4}$ and B. J. van Wees ${ }^{1}$ \\ ${ }^{1}$ Physics of Nanodevices, Zernike Institute for Advanced Materials, University of Groningen, \\ Nijenborgh 4, 9747 AG Groningen, The Netherlands \\ ${ }^{2}$ Kavli Institute of NanoScience, Delft University of Technology, 2628 CJ Delft, The Netherlands \\ ${ }^{3}$ Institute for Materials Research and WPI-AIMR, Tohoku University, 980-8577 Sendai, Japan \\ ${ }^{4}$ Université de Bretagne Occidentale, Laboratoire de Magnétisme de Bretagne CNRS, \\ 6 Avenue Le Gorgeu, 29285 Brest, France
}

(Received 19 November 2013; published 7 July 2014)

\begin{abstract}
We report the observation of the spin Peltier effect (SPE) in the ferrimagnetic insulator yttrium iron garnet (YIG), i.e., a heat current generated by a spin current flowing through a platinum (Pt)|YIG interface. The effect can be explained by the spin transfer torque that transforms the spin current in the Pt into a magnon current in the YIG. Via magnon-phonon interactions the magnetic fluctuations modulate the phonon temperature that is detected by a thermopile close to the interface. By finite-element modeling we verify the reciprocity between the spin Peltier and spin Seebeck effect. The observed strong coupling between thermal magnons and phonons in YIG is attractive for nanoscale cooling techniques.
\end{abstract}

DOI: 10.1103/PhysRevLett.113.027601

PACS numbers: 72.20.Pa, 75.78.-n, 85.80.Fi

The discovery of the spin Seebeck effect (SSE) in YIG|Pt bilayers [1] opened up a new research direction in the field of spin caloritronics. Contrary to spin-dependent thermoelectric effects carried by the electron spin-up and spindown currents that are presumably dominant in metallic ferromagnets $[2,3]$, only magnons can drive the SSE in magnetic insulators. In the SSE a temperature difference between the magnons in the magnetic insulator and the electrons in the metal contact leads to thermal pumping of a spin current [4-6]. In a suitable metal such as Pt, this spin current is transformed into an observable transverse voltage by the inverse spin Hall effect [7]. Numerical simulations of the phonon, magnon, and electron temperatures show good agreement with experiments [8]. In this Letter we report the observation of the spin Peltier effect (SPE), which is the Onsager reciprocal [9] of the SSE.

The SPE is the generation of a magnon heat current in the magnetic insulator by a spin current through the interface with the metal contact. The latter can be generated by a charge current in the Pt film that by the spin Hall effect generates a transverse spin current normal to the interface. The spin Peltier heat current generates a temperature difference between magnons and phonons in the YIG that when relaxing leads to a change in the lattice temperature. We confirm this scenario experimentally by picking up such temperature changes via proximity thermocouples. According to our modeling the experimental results are consistent with Onsager reciprocity between the SPE and the SSE, which we measure separately (see Supplemental Material [10], Sec. IV). Our results confirm recent indications for a strong magnon-phonon interaction in YIG at room temperature $[8,11,12]$.

A charge current through a Pt strip generates a transverse spin current induced by the spin Hall effect that leads to a spin accumulation $V_{s}$ at the boundaries. At the interface to YIG the spin current is absorbed as a spin transfer torque proportional to the spin mixing conductance $[13,14]$, as depicted in Fig. 1(a). When the magnetic moment of the spin accumulation $\left(\mu_{s}\right)$ at the $\mathrm{YIG} \mid \mathrm{Pt}$ interface is parallel (antiparallel) to the average magnetization direction, the spin torque transfers magnetic momentum and energy from the electrons in the Pt to the magnons in the YIG (or vice versa). Magnons are thereby annihilated (excited) [see Fig. 1(b)] leading to cooling (heating) of the magnetic order parameter [see Fig. 1(c)]. Since thermal magnons equilibrate with the lattice by magnon-phonon scattering, the nonequilibrium magnons affect the lattice temperature [see Fig. 1(b) and 1(c)] depending on the magnetization direction.

In the SSE [4] the spin current density $\left(J_{s}\right)$ pumped from the YIG into the nonmagnetic metal is proportional to the temperature difference between the magnons and electrons at the interface $\left(T_{m-e}=T_{m}-T_{e}\right)$ and the interface spin Seebeck coefficient $L_{S}, J_{s}=L_{S} T_{m-e}$. In order to arrive at a symmetric linear response matrix that reflects Onsager symmetry, the sum of the products of currents and driving forces should be proportional to the dissipation [15], leading to (see Supplemental Material [10], Sec. I)

$$
\left(\begin{array}{c}
J_{s} \\
Q_{m-e}
\end{array}\right)=\left(\begin{array}{cc}
g_{S} & L_{S} T \\
L_{S} T & \varkappa_{S}^{I} T
\end{array}\right)\left(\begin{array}{c}
V_{s} / 2 \\
T_{m-e} / T
\end{array}\right)
$$

Here we used the Onsager Kelvin relation $\Pi_{S}=S_{S} T=$ $L_{S} T / g_{S}$, where the spin Seebeck $S_{S}=\left(d V_{s} /\left(2 d T_{m-e}\right)\right)_{J_{s}=0}$ and spin Peltier $\Pi_{S}=\left(d Q_{m-e} / d J_{s}\right)_{T_{m-e}=0}$ coefficients have been defined. $g_{S}$ is the average spin conductance per unit area when spin accumulation and magnetization are collinear; i.e., the $V_{s}$ at the $\mathrm{YIG} \mid \mathrm{Pt}$ interface is either 
(a)

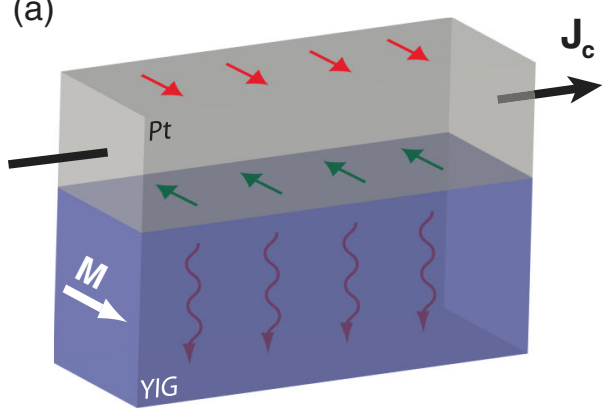

(b)

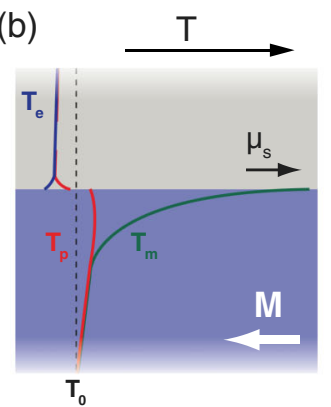

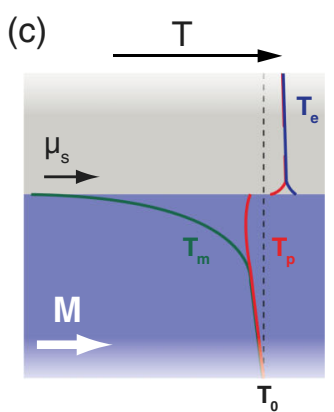

FIG. 1. (color online). Schematic figure of the spin Peltier effect at a Pt $\mid$ YIG interface. (a) A charge current through the Pt creates a transverse spin current induced by the spin Hall effect that generates a spin accumulation $V_{s}$ at the boundaries. (b) When the spin magnetic moment $\mu_{s}$ is antiparallel to $M$ the spin torque transfers angular momentum and energy from the electrons in the Pt to the magnons in the YIG thereby cooling the electrons and heating the magnons, effectively raising the magnon temperature $T_{m}$ with respect to the electron temperature $T_{e}$. (c) When $\mu_{s}$ is parallel to $M$ the spin torque transfers angular momentum and energy from the magnons in the YIG to the electrons in the Pt thereby cooling the magnons, effectively lowering $T_{m}$ with respect to $T_{e}$.

parallel or antiparallel to the average YIG magnetization. $g_{S} \approx 0.16 g_{r}$ at room temperature [16], where $g_{r}$ is the real part of the spin-mixing conductance per unit area. $\varkappa_{S}^{I}$ is the magnetic contribution to the interface heat conductance per unit area [8]. The SPE heat current density we set out to discover is therefore

$$
Q_{m-e}=L_{S} T \frac{V_{s}}{2}
$$

The devices designed for observing the SPE are fabricated on top of a $200 \mathrm{~nm}$ thick single-crystal (111) $\mathrm{Y}_{3} \mathrm{Fe}_{5} \mathrm{O}_{12}$ (YIG) film grown on a $500 \mu \mathrm{m}$ thick (111) $\mathrm{Gd}_{3} \mathrm{Ga}_{5} \mathrm{O}_{12}$ (GGG) substrate by liquid-phase epitaxy. Two temperature sensors are fabricated in close proximity to the YIG|Pt interface. The optical microscope image in Fig. 2(b) shows the $20 \times 200 \mu \mathrm{m}^{2}$ and $5 \mathrm{~nm}$ thick Pt injector film. The thermopile sensors consist of five $40 \mathrm{~nm}$ thick Pt-Constantan $\left(\mathrm{Ni}_{45} \mathrm{Cu}_{55}\right)$ thermocouples in series that are very sensitive because of the large difference in the Seebeck coefficient of these metals. In the thermopile on the right of the Pt injector the $\mathrm{Pt} \mid \mathrm{Ni}_{45} \mathrm{Cu}_{55}$ order is reversed for additional cross check measurements (see Supplemental Material [10], Sec. II). The two thermopiles and the Pt injector are connected to $5 \mid 100 \mathrm{~nm}$ thick titanium|gold contacts, providing good thermal anchoring and electrical contact to bonding pads $30 \mu \mathrm{m}$ away. All structures are patterned by electron beam lithography. The Pt injector and the $\mathrm{Ni}_{45} \mathrm{Cu}_{55}$ are deposited by dc sputtering while electron beam evaporation has been used to make the Au contacts and $\mathrm{Pt}$ thermocouple components.

An ac current is sent through the Pt injector, from $I^{+}$to $I^{-}$[Fig. 2(b)], to create $V_{s}$. The voltage over the thermopile $\left(V^{+}\right.$and $\left.V^{-}\right)$is simultaneously recorded. Using a standard lock-in detection technique the first harmonic response $(V \propto I)$ is extracted from the measured voltage. A low excitation frequency of $17 \mathrm{~Hz}$ was used to ensure a thermal steady-state condition. All measurements are carried out at room temperature.

In Fig. 2(a) the first harmonic voltage over the thermopile is shown as a function of an applied in-plane magnetic field $(B)$ for a root-mean-square current of $3 \mathrm{~mA}$ through the Pt injector. A clear switch is observed just after the

(a)

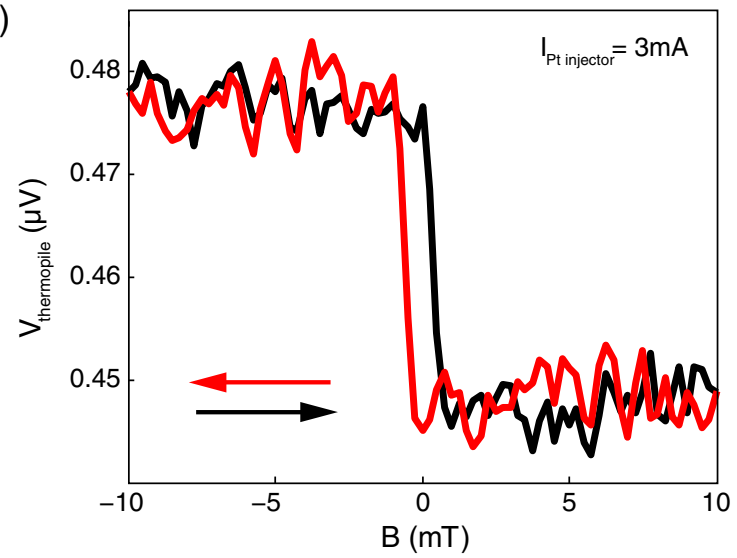

(b)

(c)
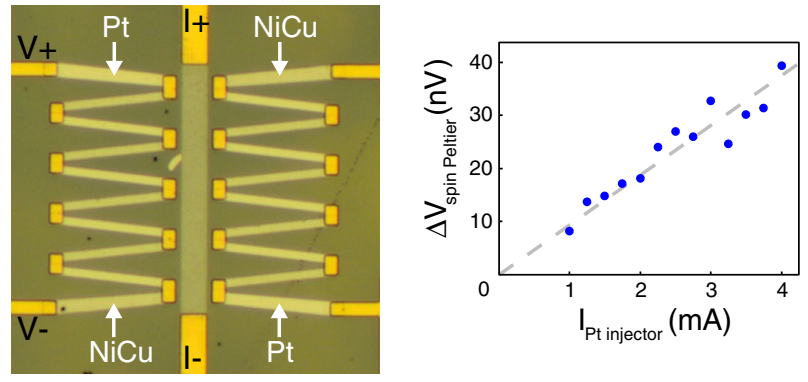

FIG. 2. (color online). (a) First harmonic voltage across the thermopile as a function of applied magnetic field. The difference between the voltage at positive and negative fields is the spin Peltier signal. (b) Optical microscope picture of the device. (c) The spin Peltier signal $\left(\Delta V_{\text {spin Peltier }}\right)$ as a function of the charge current through the Pt injector. 
applied field becomes positive, in line with the magnetization reversal of YIG at very small coercive fields [17]. The signal switches back to its original value when reversing the field with a small hysteresis. We measure a SPE signal of $33 \mathrm{nV}$ on top of a background voltage of $0.463 \mu \mathrm{V}$. We observe linear scaling of the SPE signal for currents between 1 and $4 \mathrm{~mA}$ in the Pt $\left(I_{P t \text { injector }}\right)$ [see Fig. 2(c)]. Results for four different samples (from two different batches) match the signal presented here within $15 \%$. The measurements were repeated with $B$ rotated $90^{\circ}$. No SPE signal was observed in this configuration while the background remained the same (see Supplemental Material [10], Sec. III), which confirms our interpretation.

In order to obtain quantitative information we carry out three-dimensional finite element modeling of our devices [18]. As discussed above, the SPE heat current $\left(Q_{m-e}\right)$ flows between the electron and magnon systems through the YIG|Pt interface. $Q_{m-e}$ is calculated using Eq. (2) and

$$
V_{s}=\theta J_{c} \eta \tanh \left(\frac{t}{2 \lambda}\right)
$$

where $\theta$ is the spin Hall angle, $t$ the Pt film thickness, $J_{c}$ the charge current density through the Pt injector, $\rho$ the $\mathrm{Pt}$ resistivity, $\lambda$ the spin-flip diffusion length and $\eta=2 \lambda \rho[1+$ $\left.g_{S} \rho \lambda \operatorname{coth}(t / \lambda)\right]^{-1}$ a backflow correction factor. The heat charge current densities in Pt are modelled by a three reservoir model of thermalized phonons, magnons, and electrons at temperatures $T_{p h}, T_{m}$, and $T_{e}$, respectively [8]. In linear response the charge $\left(J_{c}\right)$ and heat $(Q)$ current densities in the bulk of the materials are related to their driving forces, i.e., gradients of $\left(V, T_{p h}, T_{m}\right.$ and $\left.T_{e}\right)$ as $\vec{Q}_{x}=\kappa_{x} \vec{\nabla} T_{x}$ and

$$
\left(\begin{array}{c}
\vec{J}_{c} \\
\vec{Q}_{e}
\end{array}\right)=-\left(\begin{array}{cc}
\sigma & \sigma S \\
\sigma S T & \kappa_{e}
\end{array}\right)\left(\begin{array}{c}
\vec{\nabla} V \\
\vec{\nabla} T_{e}
\end{array}\right),
$$

where $x$ is $p h$ or $m, \sigma$ is the electrical conductivity, $S$ the Seebeck coefficient and $\kappa_{p h}, \kappa_{m}$ and $\kappa_{e}$ are the phonon, magnon and electron thermal conductivities, respectively. The interaction between the magnon and phonon subsystems in YIG and between the phonon and electron subsystems in $\mathrm{Pt}$ are taken into account by using thermal relaxation lengths, $\lambda_{m-p h}$ and $\lambda_{e-p h}$, respectively (see Supplemental Material [10], Sec. IV),

$$
\nabla^{2} T_{m-p h}=\frac{T_{m-p h}}{\lambda_{m-p h}^{2}} \quad \text { and } \quad \nabla^{2} T_{e-p h}=\frac{T_{e-p h}}{\lambda_{e-p h}^{2}}
$$

The phonon interface heat conductance $\left(\kappa_{p h}^{I}\right)$ and heat exchange between magnons and electrons across the interface $\left(\varkappa_{S}^{I}\right)$ are treated as boundary conditions [8] (see Supplemental Material [10], Sec. IV).

This model is evaluated for the material parameters listed in Table I and $g_{r}=7 \times 10^{14} \Omega^{-1} \mathrm{~m}^{-2}$ [19], $\theta=0.11$ [8],
TABLE I. Material parameters used in the model. Both $\sigma$ and $\mathrm{S}$ are measured in separate devices [20] except for $\sigma$ of the $\mathrm{Pt}$ injector, which is extracted from the SPE devices directly. $\kappa_{p h} / \kappa_{e}$ is adopted from Ref. [8] and the total $\kappa=\kappa_{p h}+\kappa_{e}$ is calculated using $\kappa=\left(\sigma / \sigma_{\text {bulk }}\right) \kappa_{\text {bulk }}$.

\begin{tabular}{lcccc}
\hline \hline & $\sigma$ & $\mathrm{S}$ & $\kappa_{p h}$ & $\kappa_{e}$ \\
\hline & $(\mathrm{S} / \mathrm{m})$ & $(\mu \mathrm{V} / K)$ & {$[\mathrm{W} /(\mathrm{m} \cdot \mathrm{K})]$} & {$[\mathrm{W} /(\mathrm{m} \cdot \mathrm{K})]$} \\
\hline $\mathrm{YIG}$ & $\ldots$ & $\ldots$ & 6 & $\cdots$ \\
$\mathrm{GGG}$ & $\ldots$ & $\ldots$ & 8 & $\ldots$ \\
$\mathrm{Au}$ & $2.7 \times 10^{7}$ & 1.7 & 1 & 179 \\
$\mathrm{Pt}$ injector & $3.5 \times 10^{6}$ & -5 & 3 & 23 \\
$\mathrm{Pt}$ thermocouple & $4.2 \times 10^{6}$ & -5 & 4 & 28 \\
$\mathrm{Ni}_{45} \mathrm{Cu}_{55}$ & $1 \times 10^{6}$ & -30 & 1 & 9 \\
\hline \hline
\end{tabular}

$\lambda=1.5 \mathrm{~nm}[8]$ and $L_{S}=7.24 \times 10^{9} \mathrm{~A} /\left(\mathrm{m}^{2} \mathrm{~K}\right)[4,8]$. The magnon heat conductivity of YIG $\left(\kappa_{m}\right)$ at room temperature is not well known so we used a $\kappa_{m}$ of $10^{-2}$ and $10^{-3} \mathrm{~W} /(\mathrm{mK})$ in order to cover the range of estimated values $[8,21]$. For YIG $\mid \mathrm{Pt}$ a $\kappa_{p h}^{I}$ of $\left.2.78 \times 10^{8} \mathrm{~W} /\left(\mathrm{m}^{2} \mathrm{~K}\right)\right)$ obtained from the acoustic mismatch model was adopted [8]. Since this model tends to overestimate the heat conductance [22], we also used $2 \times 10^{8} \mathrm{~W} /\left(\mathrm{m}^{2} \mathrm{~K}\right)$ ). In Fig. 3(a) the results are shown as a function of $\lambda_{m-p h}$. The semi transparent blue horizontal bar indicates the range of measured SPE signals that are best fitted by a $\lambda_{m-p h}$ of 0.1 to $0.2 \mathrm{~nm}$ for the ranges of $\kappa_{m}$ and $\kappa_{p h}^{I}$ discussed above.

SSE samples were fabricated and simulated by the same model and parameters used above (see Supplemental Marerial [10], Sec. V). In Fig. 3(b) the results are plotted and best fitted by $\lambda_{m-p h}$ between 0.2 and $0.5 \mathrm{~nm}$, which is consistent with the values found for the SPE, as is indeed required by Onsager reciprocity. This implies that our model captures the essential physics of the interacting electron, magnon, and phonon systems.

The observed SPE signal in Fig. 2(a) corresponds to a phonon temperature difference of $0.25 \mathrm{mK}$ at the thermopile, which according to the model is $39 \%$ of the phonon temperature difference directly at the YIG $\mathrm{Pt}$ interface. By engineering devices in which the phonon heat loss through the substrate is minimized by thinner or etched YIG films could therefore significantly enhance the measured signal. Altering the Pt injector coupling to the heat sink or placing the thermocouple on top of the Pt injector might also help.

The $\lambda_{m-p h}$ found here is smaller than the one adopted by Ref. [8] $(\approx 6 \mathrm{~nm})$ by roughly an order of magnitude. Actually, the simulations from Schreier et al. might agree better with their measurements for smaller values as well. $\lambda_{m-p h}$ extracted from Fig. 3 is quite sensitive to small variations in the modeling, which implies a large uncertainty. Nevertheless even when accepting a large error bar from 0.1 to $6 \mathrm{~nm}$ for $\lambda_{m-p h}$ we may conclude that thermal magnons and phonons interact strongly [11]. The relaxation length for thermal magnons with much higher energy 

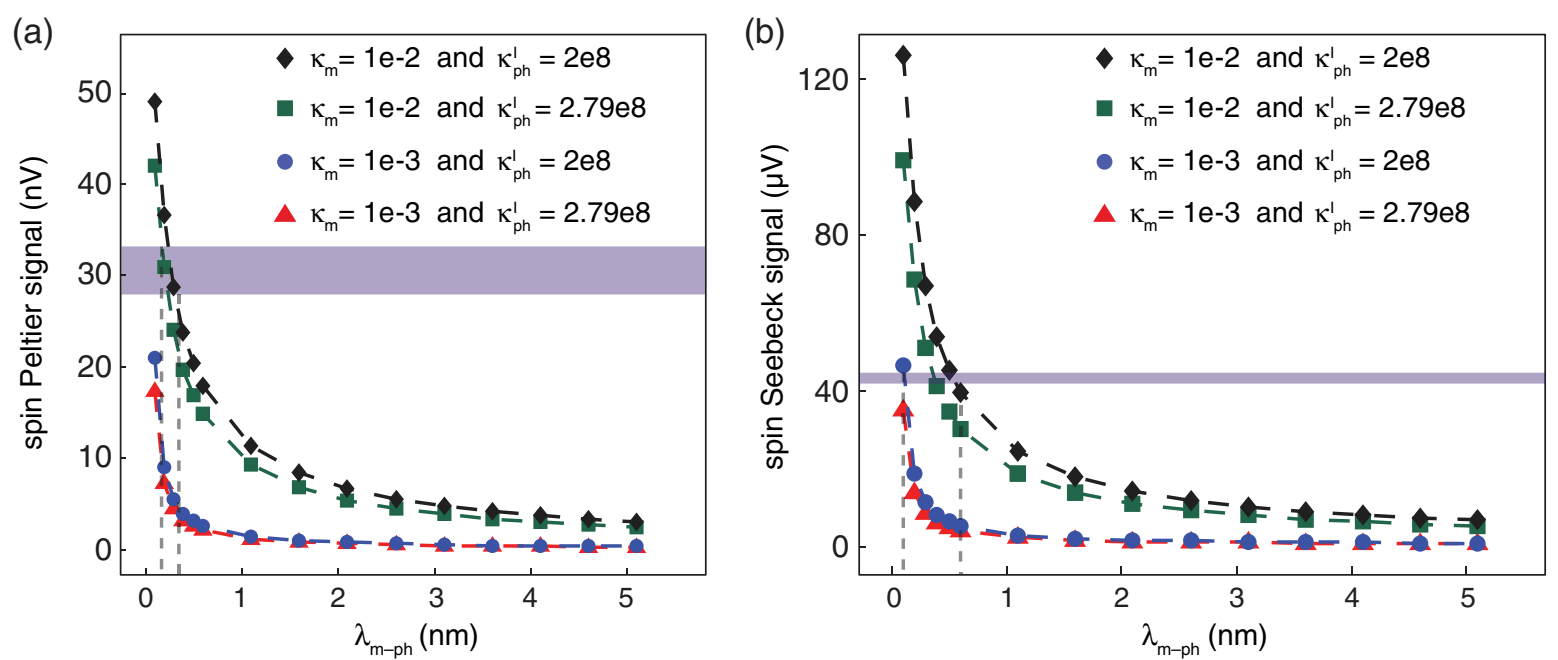

FIG. 3. (color online). The modeled SPE (a) and SSE (b) signal versus $\lambda_{m-p h}$ for two different values of $\kappa_{m}$ [W/(mK)] and two different values of $\kappa_{p h}^{I}\left[\mathrm{~W} /\left(\mathrm{m}^{2} \mathrm{~K}\right)\right]$. The semitransparent blue bar indicates the range of measured SPE and SSE effect signals.

and wave number than magnetostatic spin waves, is basically unknown. Therefore there is no evidence that the magnon-phonon interaction high up in the very complex magnon bands in YIG cannot be strong. It is also possible that most heat-spin-charge coupling takes place in the (dirty) interface region of the YIG|Pt interfaces [23]. In that case the parameters are dominated by the interface and do not reflect the bulk behavior.

The background signal in the SPE data is a factor 20 higher than we would expect from conventional charge Peltier heating and cooling at the $\mathrm{Au} \mid \mathrm{Pt}$ injector interfaces. Reference measurements on the second thermopile on the other side of the Pt injector exclude charge current leakage to the thermopile (see Supplemental Material [10], Sec. II). For an identical configuration, $\mathrm{V}^{+}$on the same side as $\mathrm{I}^{+}$, we find an opposite sign of the measured voltage, as expected for a thermal signal since the $\mathrm{Pt} \mid \mathrm{Ni}_{45} \mathrm{Cu}_{55}$ thermopile sequence is inverted. A current leak would not change sign and can therefore be excluded. The background in the second harmonic voltage is likely to be caused by the thermovoltage across the thermopile due to Joule heating in the Pt injector, since its value agrees within $17 \%$ with the modeled one. Additional measurements of frequency dependent properties (see Supplemental Material [10], Sec. VI) rule out pick-ups due to capacitive or inductive couplings.

The sign of the observed SPE and SSE signals is consistent with reciprocity. Moreover the voltage measured across the Pt detector in a RF spin pumping measurement matches the sign of the SSE voltage for the same geometry when heating the YIG relative to the $\mathrm{Pt}[24,25]$. The absolute sign of these effects has recently been determined [26].

Alternative SSE theories have been proposed by Hoffman et al. [6] and Rezende et al. [27] that do not explicitly model the phonon system and interface heat resistance. A comparison of our results with these theories would be interesting, but is beyond the scope of this current paper.

In conclusion, we report experimental proof that a spin accumulation at a Pt|YIG interface induces heat exchange between electrons and magnons on both sides. In order to knit the theory of interface transport to the observables in our experiments it is necessary to use thermal modeling. We demonstrate that for a suitable set of parameters the model can describe both SPE and SSE, but only in the presence of a strong interaction between thermal magnons and phonons in YIG. This is consistent with the results by Agrawal et al. [11] who did not detect a temperature difference between magnons and phonons. However, due to their limited spectral and spatial resolution they could only determine a not very restrictive upper bound of $0.47 \mathrm{~mm}$ for $\lambda_{m-p h}$. We hope that our results can contribute to a better understanding of coupling between thermomagnetic and thermoelectric properties. Our proof of principle opens new strategies for nanoscale cooling applications.

We would like to acknowledge B. Wolfs, M. de Roosz, and J. G. Holstein for technical assistance. This work is part of the research program of the Foundation for Fundamental Research on Matter (FOM) and supported by NanoLab NL, Marie Curie ITN Spinicur, DFG Priority Programme 1538 "Spin-Caloric Transport," Grant-in-Aid for Scientific Research (Kakenhi) 25247056/25220910, EU-FET Grant InSpin 612759, and the Zernike Institute for Advanced Materials.

*J.Flipse@ rug.nl

[1] K. Uchida, J. Xiao, H. Adachi, J. I. Ohe, S. Takahashi, J. Ieda, T. Ota, Y. Kajiwara, H. Umezawa, H. Kawai, G. E. W. Bauer, S. Maekawa, and E. Saitoh, Nat. Mater. 9, 894 (2010). 
[2] G. E. W. Bauer, E. Saitoh, and B. J. van Wees, Nat. Mater. 11, 391 (2012).

[3] J. Flipse, F. L. Bakker, A. Slachter, F. K. Dejene, and B. J. van Wees, Nat. Nanotechnol. 7, 166 (2012).

[4] J. Xiao, G. E. W. Bauer, K. C. Uchida, E. Saitoh, and S. Maekawa, Phys. Rev. B 81, 214418 (2010).

[5] H. Adachi, J. I. Ohe, S. Takahashi, and S. Maekawa, Phys. Rev. B 83, 094410 (2011).

[6] S. Hoffman, K. Sato, and Y. Tserkovnyak, Phys. Rev. B 88, 064408 (2013).

[7] A. Hoffman, IEEE Trans. Magn. 49, 5172 (2013).

[8] M. Schreier, A. Kamra, M. Weiler, J. Xiao, G. E. W. Bauer, R. Gross, and S.T.B. Goennenwein, Phys. Rev. B 88, 094410 (2013).

[9] L. Onsager, Phys. Rev. 37, 405 (1931).

[10] See Supplemental Material at http://link.aps.org/ supplemental/10.1103/PhysRevLett.113.027601, extra and more in-depth information and data supplemental to the main article, which includes Refs. [28-30].

[11] M. Agrawal, V. I. Vasyuchka, A. A. Serga, A. D. Karenowska, G. A. Melkov, and B. Hillebrands, Phys. Rev. Lett. 111, 107204 (2013).

[12] N. Roschewsky, M. Schreier, A. Kamra, F. Schade, K. Ganzhorn, S. Meyer, H. Huebl, S. Geprägs, R. Gross, and S. T. B. Goennenwein, arXiv:1309.3986.

[13] A. Brataas, G. E. W. Bauer, and P. J. Kelly, Phys. Rep. 427, 157 (2006).

[14] Z. Wang, Y. Sun, M. Wu, V. Tiberkevich, and A. Slavin, Phys. Rev. Lett. 107, 146602 (2011).

[15] H. B. Callen, Phys. Rev. 73, 1349 (1948).

[16] H. J. Jiao, J. Xiao, and G. E. W. Bauer (to be published).

[17] N. Vlietstra, J. Shan, V. Castel, B. J. van Wees, and J. Ben Youssef, Phys. Rev. B 87, 184421 (2013).
[18] COMSOL Multiphysics® 4.2a. Software for modeling and simulating physics-based systems.

[19] N. Vlietstra, J. Shan, V. Castel, J. Ben Youssef, G. E. W. Bauer, and B. J. van Wees, Appl. Phys. Lett. 103, 032401 (2013).

[20] F. L. Bakker, J. Flipse, and B. J. van Wees, J. Appl. Phys. 111, 084306 (2012).

[21] R. L. Douglass, Phys. Rev. 129, 1132 (1963).

[22] E. T. Swartz and R. O. Pohl, Rev. Mod. Phys. 61, 605 (1989).

[23] Y. Tserkovnyak (private communication).

[24] C. W. Sandweg, Y. Kajiwara, A. V. Chumak, A. A. Serga, V. I. Vasyuchka, M. B. Jungfleisch, E. Saitoh, and B. Hillebrands, Phys. Rev. Lett. 106, 216601 (2011).

[25] M. Weiler, M. Althammer, M. Schreier, J. Lotze, M. Pernpeintner, S. Meyer, H. Huebl, R. Gross, A. Kamra, J. Xiao, Y. T. Chen, H. J. Jiao, G. E. W. Bauer, and S. T. B. Goennenwein, Phys. Rev. Lett. 111, 176601 (2013).

[26] M. Schreier, G. E. W. Bauer, V. Vasyuchka, J. Flipse, K. Uchida, J. Lotze, V. Lauer, A. Chumak, A. Serga, S. Daimon, T. Kikkawa, E. Saitoh, B. J. van Wees, B. Hillebrands, R. Gross, and S. T. B. Goennenwein, arXiv:1404.3490.

[27] S. M. Rezende, R. L. Rodríguez-Suárez, R. O. Cunha, A. R. Rodrigues, F. L. A. Machado, G. A. Fonseca Guerra, J. C. Lopez Ortiz, and A. Azevedo, Phys. Rev. B 89, 014416 (2014).

[28] W. Wang and D. G. Cahill, Phys. Rev. Lett. 109, 175503 (2012).

[29] K. Uchida, H. Adachi, T. Ota, H. Nakayama, S. Maekawa, and E. Saitoh, Appl. Phys. Lett. 97, 172505 (2010).

[30] M. Weiler, M. Althammer, F. D. Czeschka, H. Huebl, M. S. Wagner, M. Opel, I. M. Imort, G. Reiss, A. Thomas, R. Gross, and S. T. B. Goennenwein, Phys. Rev. Lett. 108, 106602 (2012). 